\title{
Vitreoretinal Area
}

National Cancer Institute

\section{Source}

National Cancer Institute. Vitreoretinal Area. NCI Thesaurus. Code C157066.

The intraocular area that refers to the uvea, retina, vitreous body, and optic nerve. 